J. Clin. Chem. Clin. Biochem.

Vol. 24, 1986, pp. $369-374$

(C) 1986 by Walter de Gruyter \& Co.

Berlin - New York

\title{
Serumlipide von Alkoholikern vor und nach Abstinenz Bedeutung für das „Koronarrisiko“
}

\section{Von M. R. Clemens \\ Medizinische Klinik}

\section{H. W. Schied}

Nervenklinik und

\section{H. D. Waller}

Medizinische Klinik

Eberhard-Karls-Universität Tübingen

(Eingegangen am 16. Oktober 1985/29. Januar 1986)

Zusammenfassung: An 23 chronischen Alkoholikern wurde ein differenzierter Lipoproteinstatus bei der Aufnahme zur Entzugsbehandlung und nach 4-wöchiger Abstinenz erhoben. Neben den Cholesterin- und Triglycerid-Konzentrationen im Serum wurden mit Hilfe einer quantitativen Lipoprotein-Elektrophorese die HDL-, LDL- und VLDL-Cholesterin-Konzentrationen bestimmt. Auf Grund dieser Ergebnisse wurde das Risiko dieser Patientengruppe eingeschätzt, eine koronare Herzkrankheit zu entwickeln. Die Hälfte der Alkoholiker hatte eine Lipoprotein-Konstellation, die für ein „hohes“ oder „erhöhtes Koronarrisiko“ sprach. Dies war besonders auf erhöhte LDL-Cholesterin-Konzentrationen zurückzuführen. Durch den Alkoholenzug sank die HDL-Cholesterin-Konzentration signifikant ab, die LDL-Cholesterin-Konzentration blieb unverändert hoch. Dadurch nahm die Anzahl der Alkoholiker mit „erhöhtem Koronarrisiko“ deutlich zu.

Serum lipids of alcoholics before and after withdrawal. Importance for coronary risk

Summary: Cholesterol, triglycerides, and lipoproteins were studied in 23 alcoholics on admission for withdrawal therapy and after 4 weeks of abstinence. Lipoproteins were estimated quantitatively by lipoprotein electrophoresis. The results were used for the prediction of the risk of coronary heart disease. Half of the alcoholics had "high" or "elevated coronary risk" on admission for withdrawal. Abstinence resulted in decreased HDL-cholesterol concentrations in serum. Concurrently, the number of alcoholics with elevated coronary risk increased. This was mainly caused by high LDL-cholesterol levels on admission and after abstinence.

\section{Einführung}

Umfangreiche Untersuchungen haben gezeigt, daß Alkoholkonsum eine direkte Wirkung auf die Konzentration der Plasmalipide hat (1): Schon lange ist bekannt, daß $\not$ die Aufnahme einer größeren Menge an Alkohol zu einer transitorischen Hyperlipidämie führen $\operatorname{kann}(2,3)$. In einer Reihe von Untersuchun- gen wurde festgestellt, daß chronischer Alkoholabusus zu keiner Erhöhung des Cholesterins im Plasma führt (4-7). Triglyceride im Plasma können zwar durch eine akute Alkoholbelastung ansteigen; sie normalisieren sich jedoch wieder, wenn der Alkoholkonsum kontinuierlich fortgesetzt wird (8). Weder bei chronischen Alkoholikern $(7,9)$, noch in einer großen 
epidemiologischen Studie zur Wirkung des Alkoholkonsums auf die Blutfette (6) wurden erhöhte Triglycerid-Konzentrationen im Plasma im Vergleich mit Nichttrinkern beobachtet, sofern nicht andere Faktoren hinzugekommen waren (5).

Eine Reihe von Autoren fand bei chronischen Alkoholikern $(7,9,10)$ und Freiwilligen, die kontinuierlich größere Alkoholmengen konsumierten $(8,11,12)$, erhöhte HDL-Cholesterin-Konzentrationen im Plasma. In epidemiologischen Studien wurde eine negative Korrelation zwischen HDL-Cholesterin und koronarer Herzkrankheit gefunden (13) und dem HDL-Cholesterin eine antiatherogene Potenz zugeschrieben (14). Schon früher konnte gezeigt werden, daß mäßiger Alkoholkonsum das Risiko zur Entwicklung einer koronaren Herzkrankheit vermindern kann $(15,16)$. Andere Studien erbrachten jedoch in Bezug auf die koronare Herzkrankheit eine höhere Mortalität bei schweren Trinkern $(17,18)$.

In der vorliegenden Studie wird untersucht, wie hoch das "Koronarrisiko" bei chronischen Alkoholikern ist - eingeschätzt an Hand eines differenzierten Lipoproteinstatus (19) - und welchen Einfluß ein Alkoholentzug darauf hat.

\section{Patienten, Material und Methoden}

\section{Patienten}

Das untersuchte Kollektiv umfaßt 23 Alkoholiker (20 Männer, 3 Frauen), die in der Universitäts-Nervenklinik Tübingen zu einer 6-wöchigen Entzugsbehandlung aufgenommen wurden. 20 der 23 Alkoholiker waren zum Zeitpunkt der Untersuchung zwischen 25 und 50 Jahre alt (Altersverteilung aller Alkoholiker: 23-60 Jahre). Alle Alkoholiker tranken in den letzten 2 Jahren vor der Entzugsbehandlung regelmäßig mindestens $150 \mathrm{~g}$ Ethanol (Streuung 150 - $550 \mathrm{~g} / \mathrm{Tag}$ ). Die Mengen wurden auf Grund der Angaben berechnet, welche von den Patienten am Ende der Entzugsbehandlung gemacht wurden. Der Kontrollverlust über den Alkoholkonsum war in allen Fällen spätestens 2 Jahre vor dem Untersuchungszeitraum eingetreten. Unter den Alkoholikern waren 3 Nichtraucher (2 Männer, 1 Frau). Alle hier untersuchten Alkoholiker tranken bis mindestens 24 Stunden vor der Blutentnahme die angegebene Alkoholmenge. Sie waren in einem guten Ernährungszustand und hatten keinerlei Zeichen einer Lebercirrhose.

Die klinisch-chemischen Untersuchungen bestätigten, daß in keinem Fall ein Proteinmangel und/oder eine Leberfunktionsstörung bestand, wobei zur Beurteilung folgende Parameter bestimmt wurden und im Referenzbereich lagen: Bilirubin, Albumin, Quick-Test, Cholinesterase. Alle Alkoholiker hatten über die Norm erhöhte Aktivitäten der $\gamma$-Glutamyltransferase und Erythrocyten mit einem erhöhten mittleren korpuskulären Volumen."

Material

Das Blut wurde morgens zwischen 7 und $8 \mathrm{Uhr}$ nach einer mindestens 12-stündigen Fastenperiode entnommen.
Methoden

HDL-, VLDL- und LDL-Cholesterin wurden durch eine quantitative Lipoproteinelektrophörese (Lipidophor All-In, Immuno Diagnostik, Heidelberg) bestimmt. Ein ausführlicher Vergleich zwischen der angewandten Methode und der. Bestimmung von HDL-, LDL- und VLDL-Cholesterin mittels Ultrazentrifugation wurde kürzlich publiziert: Dabei konnte gezeigt werden, daß die Lipoproteinelektrophorese für die Quantifizièrung der einzelnen Cholesterinfraktionen sehr gut geeignet ist und ihr eine hohe Aussagekraft bei der Identifizierung von Koronarkranken zukommt.

Sämtliche anderen Bestimmungen wurden mit Routinemethoden (Zentrallabor der Medizinischen Klinik Tübingen) durchgeführt.

\section{Ergebnisse}

Bei der Aufnahme zur Entzugsbehandlung (A) und nach 4-wöchiger Abstinenz (B) wurden die Plasmalipide von 23 Alkoholikern untersucht.

In 11 Fällen lag die Cholesterin- und in 4 Fällen die Triglycerid-Konzentration bei der ersten Untersuchung (A) über der Norm. Nach 4-wöchiger Abstinenz (B) war nur noch bei 3 Alkoholikern die Cholesterin-Konzentration erhöht; die Triglycerid-Konzentrationen waren in 7 Fällen über der Norm. Kombinierte Hypercholesterin- und Hypertriglyceridämien hatten 3 Patienten bei der Aufnahme und nur noch einer nach 4-wöchiger Abstinenz (Abb. 1).

Der Mittelwert \pm Standardabweichung der Cholesterin-Konzentration im Serum betrug bei der Aufnahme $6,27 \pm 1,40 \mathrm{mmol} / \mathrm{l}$ (Referenzbereich: 3,89-6,48 mmol/1), nach 4-wöchiger Abstinenz war er signifikant auf $5,70 \pm 0,85 \mathrm{mmol} / 1$ abgefallen ( $p<0,01$ im gepaarten t-Test). Der Mittelwert der Triglyceride betrug bei Aufnahme 1,21 $\pm 0,63 \mathrm{mmol} / 1$ (Referenzbereich: $0,84-1,94 \mathrm{mmol} / \mathrm{l}$ ) und war nach 4-wöchiger Abstinenz auf 1,75 $\pm 0,79 \mathrm{mmol} / 1$ angestiegen ( $p<0,01$ im gepaarten $t=$ Test) (Abb. 1).

HDL-Cholesterin-Konzentrationen lagen anfänglich bei 17 Alkoholikern über $1,30 \mathrm{mmol} / 1$, davon in 5 Fällen über 2,1 mmol/1. Nach 4-wöchiger Abstinenz wiesen nur noch 4 Alkoholiker HDL-Werte über 1,30 $\mathrm{mmol} / \mathrm{l}$ auf, wohingegen Werte über $2,1 \mathrm{mmol} / 1$ in keinem Fall mehr gemessen wurden (Abb. 2). Der Mittelwert des HDL-Cholesterins betrug bei der Aufnahme 1,74 $\pm 0,70 \mathrm{mmol} / \mathrm{l}$. Er lag nach 4-wöchiger Abstinenz signifikant niedriger $(0,98 \pm 0,34 \mathrm{mmol} / \mathrm{l}$; $\mathrm{p}<0,01)$.

Bei 10 Alkoholikern lag die LDL-Cholesterin-Konzentration bei der Aufnahme über 4,92 mmol/1, nach 4-wöchiger Abstinenz nur noch in 77 Fällen (Abb. 3). 


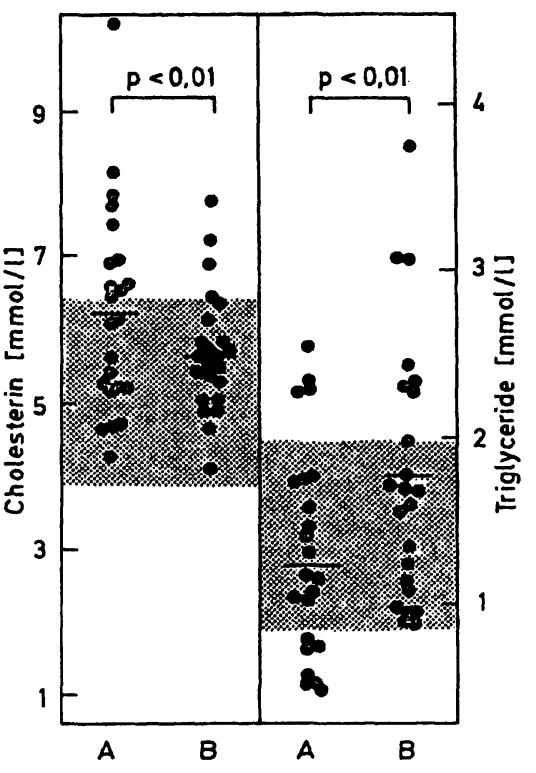

Abb. 1. Cholesterin- und Triglycerid-Konzentrationen im Serum von Alkoholikern bei Aufnahme zur Entzugsbehandlung (A) und nach 4-wöchiger Abstinenz (B). Punktierte Flächen $=$ Referenzbereiche. Berechnung der Signifikanzen mit dem gepaarten t-Test.

Der Mittelwert des LDL-Cholesterins betrug 4,40 \pm $1,53 \mathrm{mmol} / 1$ bei Aufnahme und wies nach 4-wöchiger Abstinenz keine signifikante Änderung auf $(4,53 \pm$ $1,01 \mathrm{mmol} / \mathrm{l})$.

Der Quotient LDL/HDL-Cholesterin (mol $\cdot 1^{-1} / \mathrm{mol}$ $\cdot 1^{-1}$ ) lag bei 6 Alkoholikern bei Aufnahme (A) (Abb. 4) über 4 (Mittelwert der 23 Alkoholiker: 3,2 $\pm 2,1$ ),

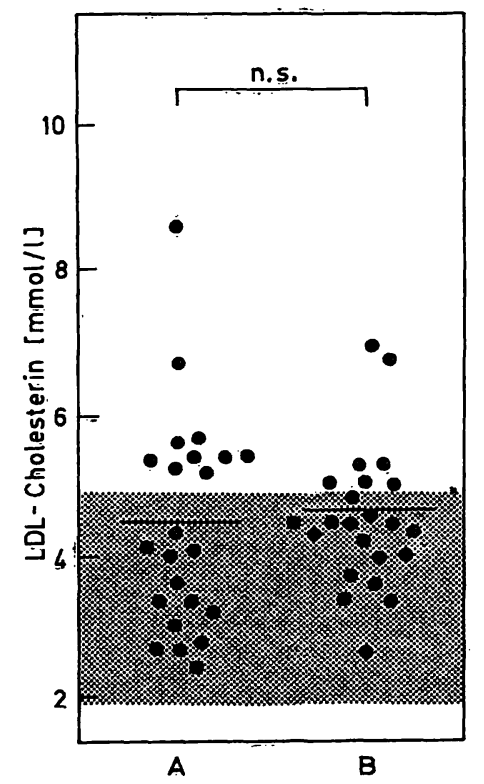

Abb. 3. LDL-Cholesterin-Konzentrationen im Serum von Alkoholikern bei Aufnahme zur Entzugsbehandlung (A) und nach 4-wöchiger Abstinenz (B).

Punktierte Fläche $=$ Referenzbereich. Berechnung der Signifikanz mit dem gepaarten $\mathrm{t}$-Test.

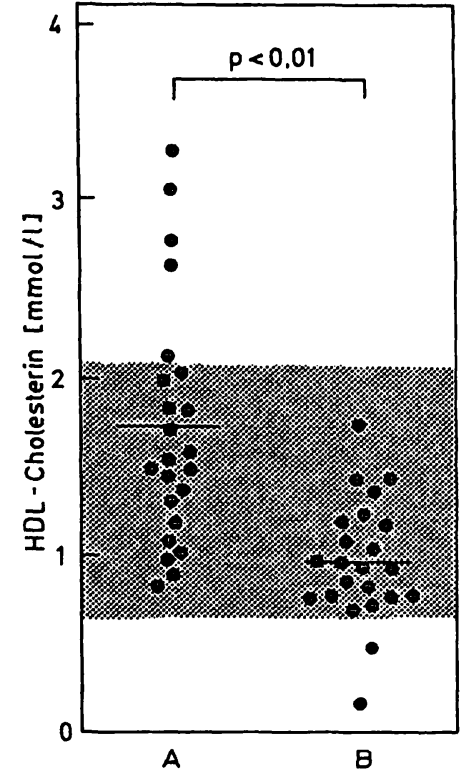

Abb. 2. HDL-Cholesterin-Konzentrationen im Serum von Alkoholikern bei der Aufnahme zur Entzugsbehandlung (A) und nach 4-wöchiger Abstinenz (B).

Punktierte Fläche $=$ Referenzbereich. Berechnung der Signifikanz mit dem gepaarten t-Test.

davon in einem Fall gleichzeitig mit einem LDLCholesterinwert zwischen 3,63 und 4,66 mmol/1. Nach 4-wöchiger Abstinenz (B) war der Quotient bei der Mehrzahl der Alkoholiker angestiegen (18 von 23 Fälle). Dabei lag er bei 15 der 23 Kontrollbestimmungen über $4(5,4 \pm 2,9 ; A$ vs. B: $p<0,01$; Abb. 4$)$; in 8 Fällen wurde zusätzlich eine LDL-Cholesterin zwischen 3,63 und $4,66 \mathrm{mmol} / 1$ gemessen.

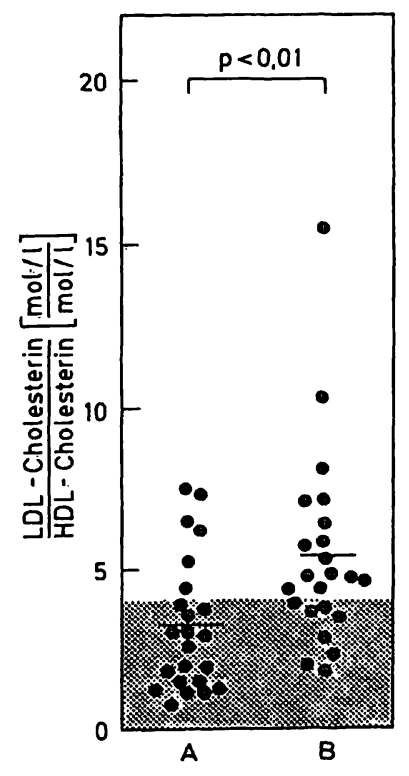

Abb. 4. Quotient LDL/HDL-Cholesterin von Alkoholikern bei Aufnahme zur Entzugsbehandlung (A) und nach 4wöchiger Abstinenz (B).

Punktierte Fläche $=$ Referenzbereich. Berechnung der Signifikanz mit dem gepaarten t-Test. 
Die Konzentrationen des VLDL-Cholesterins lagen bei der Aufnahme bei allen Alkoholikern unter der oberen Normgrenze von $0,65 \mathrm{mmol} / 1$ (Abb. 5). Nach 4-wöchiger Abstinenz war keine signifikante Veränderung der mittleren VLDL-Cholesterinkonzentration festzustellen, wobei in einem Fall eine Konzentration von $0,80 \mathrm{mmol} / 1$ gemessen wurde.

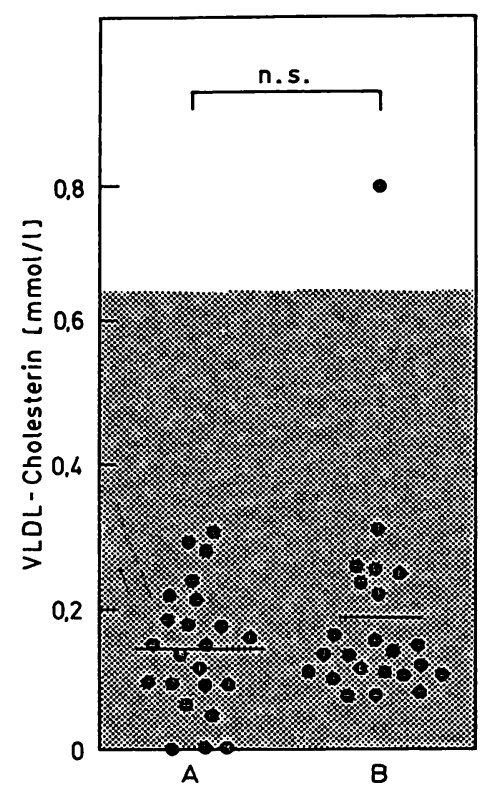

Abb. 5. VLDL-Cholesterin-Konzentrationen im Serum von Alkoholikern bei Aufnahme zur Entzugsbehandlung (A) und nach 4-wöchiger Abstinenz (B).

Punktierte Fläche $=$ Referenzbereich. Berechnung der Signifikanz mit dem gepaarten t-Test.

Das „Koronarrisiko“ wurde nach Cremer et al. eingeschätzt (Tab. 1). Bei 10 von 23 Alkoholikern bestand ein sog. „hohes Risiko“ bei der Aufnahme zur Entzugsbehandlung. Nach 4-wöchiger Abstinenz war zwar bei vielen Alkoholikern das als antiatherogen geltende HDL-Cholesterin abgefallen, trotzdem bestand ein sog. "hohes Risiko" nur noch bei 7 von 23 Alkoholikern. Demgegenüber lag die Anzahl der Alkoholiker mit einem sog. „erhöhten Risiko“ nach 4-wöchiger Abstinenz höher als bei der Aufnahme.

Tab. 1. „Koronarrisiko“ von Alkoholikern.

\section{Hohes Risiko}

(LDL-Cholesterin > 4,66 mmol/l)

10 von 23 Alkoholikern bei Aufnahme

7 von 23 Alkoholikern nach 4-wöchiger Abstinenz.

zusätzlich

erhöhtes Risiko

(LDL/HDL-Quotient $>4$

und LDL-Cholesterin 3,63-4,66 mmol/l)

1 von 23 Alkoholikern bei Aufnahme

8 von 23 Alkoholikern nach 4-wöchiger Abstinenz

\section{Diskussion}

Auf Grund der heute vertretenen pathophysiologischen Vorstellungen zur Atherogenese kann man annehmen, daß der Quantifizierung der einzelnen Lipoproteinfraktionen des Serums eine größere Bedeutung für die Früherkennung Koronargefährdeter zukommt als der reinen Bestimmung des Cholesterins und der Triglyceride.

Die LDL und das von ihnen transportierte Cholesterin gilt allgemein als der entscheidende Risikofaktor der Koronarsklerose $(23-25)$. Hingegen ist die Bedeutung der VLDL für die Atherogenese umstritten $(26,27)$, wobei es Hinweise darauf gibt, daß abnorm zusammengesetzte VLDL in der Atherogenese bedeutsam sind $(23,28)$.

HDL-Partikel können wahrscheinlich im Gewebe abgelagertes Cholesterin mobilisieren und zur Leber zurücktransportieren. Weiterhin scheinen HDL-Partikel die LDL-abhängige Cholesterinzuufuhr ins Gewebe behindern zu können. Auf diese Befunde wird zum Teil die „antiatherogene“ Wirkung der HDL (14, $25,29)$ zurückgeführt.

Da einerseits durch epidemiologische Studien die hohe Aussagekraft einer kompletten Lipoproteinbestimmung, verglichen mit der Quantifizierung einzelner Lipoproteine, gezeigt werden konnte (30), und andererseits dem Alkoholkonsum eine antiatherogene Wirkung zugeschrieben wurde $(6,16,31)$, untersuchten wir die Lipoprotein-Zusammensetzung des Serums von Alkoholikern vor. und nach einer Abstinenzperiode.

In Übereinstimmung mit den Angaben in der Literatur fanden auch wir im Serum der bier untersuchten Alkoholiker relativ hohe HDL-Cholesterin-Konzentrationen $(7-12)$. In über $70 \%$ der Fälle konnten bei der Aufnahme von Alkoholikern zur Entzugsbehandlung HDL-Cholesterin-Konzentrationen über 1,30 mmol/l gemessen werden. Trotz dieses Befundes konnten diese Alkoholiker nicht als Patienten mit niedrigem Koronarrisiko eingeschätzt werden, da in nahezu 50\% der Fälle neben den hohen HDL-Cholesterin-Konzentrationen auch hohe LDL-CholesterinKonzentrationen gemessen wurden. Andere Untersucher fanden bei Alkoholikern niedrigere LDL-Cholesterin-Konzentrationen als bei gesunden Kontrollpersonen $(32,33)$.

Nach 4-wöchiger Alkoholkarenz war die Żahl der Alkoholiker mit einem "hohen Koronarrisiko" nicht signifikant höher als bei der Aufnahme zur Entzugsbehandilung, jedoch stieg die Zahl der Alkoholiker mit einem „erhöhten Koronarrisiko" signifikant an. Dies war in erster Linie durch die verminderten HDĹ- 
Cholesterin-Konzentrationen und dem daraus resultierenden höheren Quotienten LDL/HDL-Cholesterin nach der 4-wöchigen Abstinenzperiode zu erklären.

Durch den Entzug des Alkohols kam es zu einem Anstieg der Triglyceride. Dies wurde auch von anderen Untersuchern beobachtet $(9,32,33)$ und ist wahrscheinlich darauf zurückzuführen, daß sich durch den Alkoholentzug die Lipoproteinlipase-Aktivität normalisiert. Dieses Enzym ist bei chronischem Alkoholabusus erhöht, sofern die Leberfunktion ungestört ist (8), und führt zu einem gesteigerten Umsatz der Triglyceride im Plasma $(8,9)$. Im Gegensatz zu den Triglyceriden nahm die Cholesterin-Konzentration im Serum der Alkoholiker durch die Alkoholkarenz ab. Dies kann vorwiegend auf die verminderte HDLFraktion zurückgeführt werden.

Wie sind die relativ hohen LDL-Cholesterinwerte bei den hier untersuchten Alkoholikern zu erklären? In einer früheren Untersuchung (43) konnten wir zeigen, $\mathrm{da} ß$ die hier untersuchten Alkoholiker eine stark verminderte Linolsäure-Konzentration im Plasma vor Beginn der Abstinenzperiode aufwiesen. Ein Linolsäuremangel bei chronischem Alkoholismus wurde auch von anderen Untersuchern beobachtet $(34,35)$. Er kann Folge einer verminderten Zufuhr, einer Resorptionsstörung $(36,37)$, eines vermehrten Bedarfs durch eine gesteigerte Prostaglandin-Biosynthese (38, 39) und einer durch freie Radikale bedingten erhöhten Synthese eines Isomers der Linolsäure (Octadeca9,11-diensäure) sein (40).

Dem Linolsäuremangel kann bei der Entstehung der erhöhten LDL-Cholesterin-Konzentrationen eine wesentliche Bedeutung zukommen. So ist ein Zusammenhang zwischen Linolsäurezufuhr und Cholesterin-Metabolismus schon lange bekannt (41). Die $\mathrm{Zu}$ fuhr von Linolsäure führt $z$. B. zu einer Verminderung des LDL-Cholesterins (42). Auch wurde festgestellt, $\mathrm{da} ß$ eine niedrige Linolsäurezufuhr (gemessen als Anteil der Linolsäure im Plasma bzw. Erythrocytenmembran) das Risiko zur Entwicklung einer koronaren Herzkrankheit bzw. eines Myokardinfarktes deutlich erhöht $(20-22)$.

\section{SchluBfolgerungen}

Auf Grund der vorliegenden Ergebnisse muß davon ausgegangen werden, daß bei Alkoholikern ein höheres Koronarrisiko als bei Gesunden besteht. Hierbei könnte der von uns beobachtete Linolsäuremangel bei Alkoholikern von Bedeutung sein. Alkoholkonsum führt - im Gegensatz zu empirischen Angaben - zu keiner Verminderung des Koronarrisikos bei den hier untersuchten Alkoholikern.

\section{Literatur}

1. Lieber, C. S., Leevy, C. M., Stein, S. W., George, W. S., Cherrick, C. R., Abelmann, W. M. \& Davielson, C.S. (1962) J. Lab. Clin. Med. 59; 826-832.

2. Jones, P. D., Loskowsky, M. S., Davidson, C. S. \& Lieber, C. S. (1963) J. Lab. Clin. Med. 62, 675-682.

3. Loskoswsky, W. S., Jones, D. P., Davidson, C. S. \& Lieber, C. S. (1963) Am. J. Med. 35, 794-803.

4. Avogaro, P. \& Cazzolato, G. (1975) Metabolism 24, $1231-1242$.

5. Böttiger, L. E., Carlson, L. A., Hultman, E. \& Romanns, V. (1976) Acta Med. Scand. 199, 357-361.

6. Castelli, W. P., Doyle, J. T., Gordon, T., Hames, C. G., Hjortland, M. C., Hulleỳ, S. B., Kagan, A. \& Zukel, W. J. (1977) Lancet $I I, 153-155$.

7. Danielsson, B., Ekman, R., Fex, G., Johansson, B. G., Kristensson, H., Nilson-Ehle, P. \& Wadstein, J. (1978) J. Clin. Lab. Invest. 38, 113-119.

8. Belfrage, P., Berg, B., Hägerstrạnd, I., Nilsson-Ehle, P., Tornqvist, H. \& Wiebe, T. (1977) Eur. J. Clin. Invest. 7, $127=131$.

9. Weidman, S. W., Beard, J. D. \& Sabesin, S. M. (1984) Atherosclerosis $52,151-166$.

10. Johansson, B. G. \& Medhus, A. (1974) Acta Med. Scand. $195,273-277$.

11. Glueck, C. J., Hogg, E., Allen, C. \& Gartside, P. S. (1980) Am. J. Clin. Nutr. 33, $2287-2293$.

12. Crouse, J. R. \& Grundy, S. M. (1984) J. Lipid Res. 25, $486-496$.

13. Heiss, G., Johnson, N. J., Reiland, S., Davis, C. E. \& Tyroler, H. A. (1980) Circulation 62 (Suppl. IV) 116-136.
14. Miller, N. E. (1978) Lipids 13, 914-919.

15. Klatsky, A. L., Friedman, G. D. \& Siegelaub, A. B. (1974) Ann. Intern. Med. 81, 294-301.

16. Yano, K., Rhoads, G. G. \& Kagan, A. (1977) N. Engl. J. Med. 297, 405-409.

17. Dyer, A. R., Stamler, J., Paul, O., Berkson, D. M., Lepper, M. H., McKean, H., Shekelle, R., Lindberg, H. A. \& Garside, D. (1977) Circulation 50, 1067.

18. Kozararevic, D., McGee, D., Vojvodic, N., Racic, Z., Dawber, T., Gordon, T. \& Zukel, W. (1980) Lancet I, 613-616.

19. Cremer, P., Seidel, D. \& Wieland, H. (1985) Lab. Med. 9, $39-51$.

20. Simpson, H. C. R., Barker, K., Carter, R. D., Cassels \& Mann, J. J. (1982) Br. J. Med. 285, 683-684.

21. Miettinen, T. A., Naukkarinen, V., Huttunen, J. K., Mattila, S. \& Kumlin, T. (1982) Br. J. Med. 285, 993-996.

22. Valek, J., Hammer, J., Kohout, M., Grafnetter, D., Vondra, K. \& Topinka, V. (1985) Atherosclerosis 54, 111-118.

23. Brown, M. S. \& Goldstein, J. C. (1983) J. Clin. Invest. 72, $743-747$.

24. Ross, R. \& Glomset, J. A. (1976) N. Engl. J. Med. 295, $369-377$.

25. Lewis, B. (1983) Br. Med. J. 287, 1161-1163.

26. Kannel, W. B. (1976) Am. J. Cardiol. 37, 268-282.

27. Heyden, S., Heiss, G., Bartel, A. \& Hames, C. G. (1980) J. Chron. Dis. 33, 275-282.

28. Assmann, G. (1982) Lipidstoffwechsel und Atherosklerose. Schattauer Verlag, Stuttgart, New York.

29. Assmann, G., Schriewer, H., Schulte, H. \& Oberwittler, W. (1980) Internist 21, 202-212. 
30. Cremer, P., Weise, M., Wieland, H., Seidel, D., Schulze, F. \& Kreuzer, H. (1982) Innere Medizin 9, 257-265.

31. Barboriak, J. J., Anderson, A. J. \& Hoffmann, R. G. (1979) J. Lab. Clin. Med. 94, 348-353.

32. Ekman, R., Fex, G., Johansson, B. G., Nilsson-Eble, P. \& Wadstein, J. (1981) Scand. J. Clin. Lab. Invest. 41, 709-715.

33. Taskinen, M. R., Välimäki, M., Nikkilä, E., Kuusi, T., Ehnholm, C. \& Ylikahri, R. (1982) Metabolism 11, 1168-1174.

34. Alling, C., Aspenström, G., Dencker, S. J. \& Svennerholm, L. (1979) Acta Med. Scand. (Suppl.) 631, 1-38.

35. Alling, C., Gustavsson, L., Kristensson-Aas, A. \& Wallersted, S. (1984) Scand. J. Clin. Lab. Invest. 44, 283-289.
36. Mezey, E., Jow, E., Slavin, R. E. \& Tabon, F. (1970) Gastroenterology $59,657-664$.

37. Lindenbaum, J. \& Lieber, C. S. (1975) Ann. N. Y. Acad. Sci. $252,228-234$.

38. Alling, C., Becker, W., Jones, A. W. \& Änggärd, E. (1984) Clin. Exp. Res. 8, 238-242.

39. Landolfi, R. \& Steiner, M. (1984) Blood 64, 679-682.

40. Fink, R., Clemens, M. R., Marjot, D. H., Patsalos, P., Cawood, P., Norden, A. G., Iversen, S. A. \& Dormandy, T. L. (1985) Lancet II, $291=294$.

41. Zöllner, N. \& Keller, C. (1984) Internist 25, 261 - 267.

42. Packard, C. J. \& Shepherd, J. (1985) Klin. Wochenschr. 63, 344-351.

43. Clemens, M. R., Schied, H. W., Daiss, W. \& Waller, H. D. (1986) Klin. Wochenschr. 64, $181-185$.

Dr. M. R. Clemens

Medizinische Klinik

Abt. Innere Med. II

Otfried-Müller-Str. 10

D-7400 Tübingen 1 